\title{
EXTRACORPOREAL ELIMINATION OF CIRCULATING PEGYLATED LIPOSOMAL DOXORUBICIN (PLD) TO ENHANCE THE BENEFIT OF CYTOSTATIC THERAPY IN PLATINUM-RESISTANT OVARIAN CANCER PATIENTS
}

\author{
Ondřej Kubeček ${ }^{1}$, Milan Bláha ${ }^{2}$, Daniel Diaz-Garcia ${ }^{3}$,Stanislav Filip ${ }^{I}$ \\ Department of Oncology and Radiotherapy, Charles University in Prague, Medical Faculty and University Hospital in Hra- \\ dec Králové, Czech Republic '; 4th Department of Internal Medicine - Haematology, Charles University in Prague, Medical \\ Faculty and University Hospital in Hradec Králové, Czech Republic²; Department of Histology and Embryology, Charles \\ University in Prague, Medical Faculty and University Hospital in Hradec Králové, Czech Republic ${ }^{3}$
}

Summary: Ovarian cancer is the fifth most common malignancy in the world's female population and with the highest lethality index among gynecological tumors. The prognosis of metastatic disease is usually poor, especially in platinum-resistant cases. There are several options for the treatment of metastatic disease resistant to platinum derivates (e.g. paclitaxel, topotecan and pegylated liposomal doxorubicin), all of which are considered equipotent. Pegylated liposomal doxorubicin (PLD) is a liposomal form of the anthracycline antibiotic doxorubicin. It is characterized by more convenient pharmacokinetics and a different toxicity profile. Cardiotoxicity, the major adverse effect of conventional doxorubicin, is reduced in PLD as well as hematotoxicity, alopecia, nausea and vomiting. Skin toxicity and mucositis, however, emerge as serious issues since they represent dose and schedule-limiting toxicities. The pharmacokinetics of PLD (prolonged biological half-life and preferential distribution into tumor tissue) provide new possibilities to address these toxicity issues. The extracorporeal elimination of circulating liposomes after PLD saturation in the tumor tissue represents a novel and potent strategy to diminish drug toxicity. This article intends to review PLD characteristics and the importance of extracorporeal elimination to enhance treatment tolerance and benefits.

Keywords: Pegylated liposomal doxorubicin; Palmar-plantar erythrodysesthesia; Rheopheresis, Toxicity

\section{Introduction}

Malignant ovarian tumors are the fifth most common malignancy, and the fourth leading cause of death by malignant disease in the world's female population (1) as they possess the highest lethality index amongst gynecological tumors (2). More than $70 \%$ of patients are diagnosed at a late stage (3). Most ovarian tumors are of epithelial origin (approximately $90 \%$ ) with invasive serous carcinoma being the most common histological type (4). Non-epithelial tumors represent less than $10 \%$ of ovarian tumors, with a different prognosis and treatment.

The therapeutic strategies of epithelial ovarian tumors are based on the combination of surgery and chemotherapy. Tumor debulking with minimal residual tumor mass, followed by platinum-based chemotherapy, are considered standard procedure for advanced ovarian cancer (2). The most common regimen in first-line therapy is the combination of paclitaxel $175 \mathrm{mg} / \mathrm{m}^{2}$ and carboplatin AUC 5-6 administered every 21 days. Although the response rate (RR) for this combination in advanced disease exceeds $80 \%$ and a complete response is achieved in $40-60 \%$, most patients tend to relapse within a median progression-free survival of 18 months $(2,5)$. The patients' prognosis and the probability of attaining a response to second and subsequent chemotherapy lines, depends strongly on the time interval defined by the last dose of preceding line of chemotherapy and the time of progression, e.g. platinum-free interval (1). Patients are divided into four groups according to this time interval, affecting both prognosis and further treatment strategy. Patients progressing during the therapy or within 4 weeks after the last dose are defined as platinum-refractory. Patients with disease progression within 6 months are defined as platinum-resistant, and partially platinum-sensitive patients present disease progression between 6 and 12 months after cessation of preceding chemotherapy line. The most favorable prognostic group of platinum-sensitive patients is defined by disease progression beyond 12 months from the last dose of platinum-based chemotherapy (6). The treatment of platinum-sensitive patients should be based on a combined therapy containing a platinum derivate (carboplatin or cisplatin) $(1,7)$. However, platinum-resistant and 
platinum-refractory patients in particular, do no benefit from further platinum-based chemotherapy treatments. The prognosis of this patient subgroup is generally poor with overall survival (OS) of less than 12 months (1). The treatment of these patients should therefore be focused on sustaining the best possible quality of life and symptom control (1). Sequential single-agent therapy using non-platinum-based agents is generally recommended for these patients, as a combined therapy strategies are not an improvement over a single-agent therapy (1). The most common regimens include docetaxel, topotecan, weekly or 3-weekly paclitaxel, gemcitabine or pegylated liposomal doxorubicine (PLD). The response rate (RR) to these agents is similar, with variations from $17 \%$ to $27 \%$ and lacking superiority between them $(1,7)$. The main difference between these agents is found within their toxicity profile. Therefore, the selection of the therapeutic treatment should be based on toxicity, clinical situation and preferences of each individual patient (1).

\section{Pegylated liposomal doxorubicin (PLD)}

Pegylated liposomal doxorubicin (PLD) is a formulation based on the anthracycline antibiotic doxorubicin, encapsulated in polyethylene-glycol (PEG) coated liposomes (8). Liposomes are small vesicles composed of a lipid bilayer membrane with an aqueous core (Fig. 1) with an average size ranging from a few nanometers to several micrometers (9). The approximate mean size of PLD is $90 \mathrm{~nm}(10,12)$. The surface of the liposomes is covered with a dense layer of PEG covalently attached to the lipid membrane in a process called pegylation (11). This formulation leads to a significant change in the toxicity profile and pharmacokinetic properties of the parent drug. Cardiotoxicity, the limiting adverse effect of conventional doxorubicin, is significantly reduced in PLD (12). The polyethylene-glycol coating protects serum opsonins from binding to the liposome surface, decreasing liposome degradation by the reticuloendothelial system (RES) (11). This mechanism is responsible for the significantly increased biological half-life $\left(T_{1 / 2}\right)$ of PLD as compared to free doxorubicin (11). PLD is currently approved for the treatment of AIDS-related Kaposi's sarcoma,

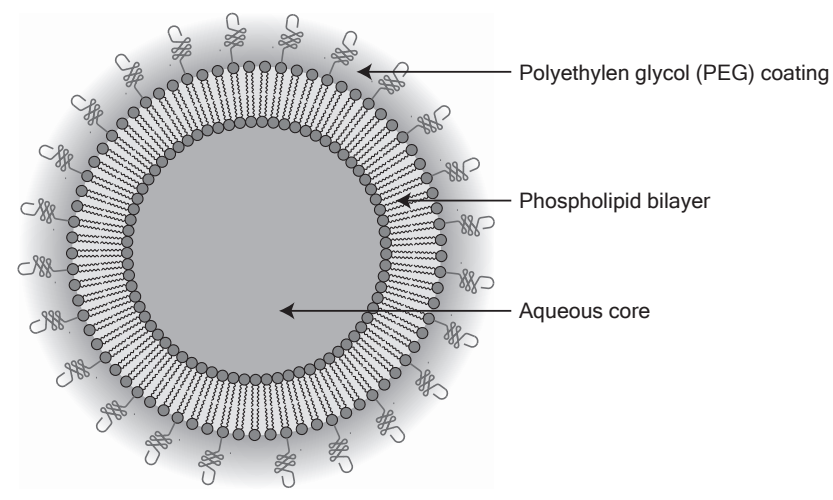

Fig. 1: Schematic diagram of pegylated liposome. metastatic breast cancer, multiple myeloma, and metastatic ovarian cancer (8). The standard regimen for the treatment of platinum-resistant ovarian cancer is $50 \mathrm{mg} / \mathrm{m}^{2}$ intravenously every 28 days until progression or serious toxicity are found (13). PLD is available under the trademark name Doxil ${ }^{\circledR}$ (US) or Caelyx ${ }^{\circledR}$ (outside US) (14).

\section{Mechanism of action}

The active agent of PLD is doxorubicin hydrochloride (8). Doxorubicin (DOX) is an anthracycline antibiotic originally isolated from Streptomyces peucetius (15). Anthracyclines are some of the most potent and commonly used anti-cancer drugs that have been produced so far (16). Their chemical structure consists of two major parts: tetracyclic aglycon (in the case of DOX, it is called doxorubicinon), a chromophore responsible for the typical red color of anthracyclines, and the amino sugar daunosamine (Fig. 2) (15). The polycyclic aglycon is essential for the intercalation of DOX between DNA strands, whereas the amino sugar (bearing a positive charge in most cellular compartments) stabilizes the intercalation by electrostatic interactions with the negatively charged phosphate groups in the DNA chain (12). This DNA intercalation is responsible for the deformation of the DNA double helix, and topoisomerase II inhibition through stabilization of otherwise reversible complexes between DNA and topoisomerase II (anthracycline-DNA-topoisomerase II ternary complexes) $(15,16,17)$. The fully functional activity of this enzyme is necessary for DNA replication, transcription, recombination and chromatin remodeling. Its activity consists in creating transient DNA breaks that are consecutively released after changing the conformation of the DNA(15). When inhibiting topoisomerase II, the ternary DNA-topoisomerase complex is stabilized and DNA release becomes impaired. This results in the creation of permanent double-strand DNA breaks with a lethal effect to the affected cells (15). Production of reactive oxygen species (ROS) is considered another important mechanism of DOX anti-tumour activity (18). ROS can cause direct damage to the DNA, RNA, lipids and proteins, and is also responsible for cardiotoxicity, the major adverse effect of DOX (19).

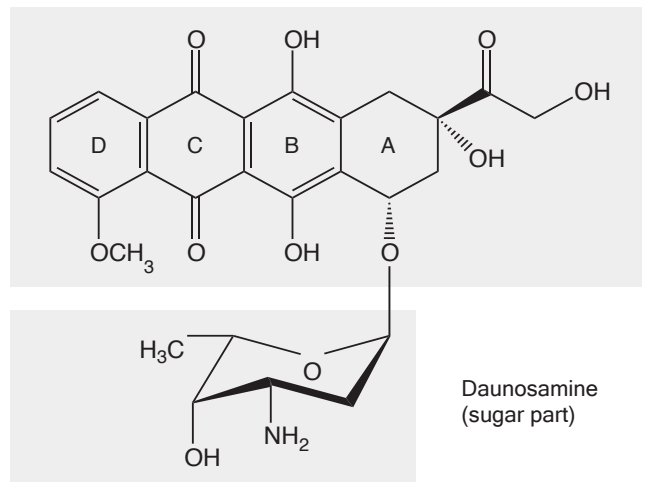

Doxorubicinon (aglycone)

Fig. 2: Structural formula of doxorubicin molecule. 


\section{Adverse effects, toxicity}

The toxicological profile of PLD is quite different from that of free DOX, with palmar-plantar erythrodysesthesia (PPE, also called hand-foot syndrome) and mucositis being the most common adverse effects (20). Although not life-threatening, these adverse effects might, in severe cases, lead to dose reduction or chemotherapy cycle postponement, negatively influencing therapy outcome (21).

PPE incidence can reach $50 \%$ for any grade and 20\% for grades 3 and 4 (using standard regimen $50 \mathrm{mg} / \mathrm{m}^{2}$ every 28 days) $(22,23)$. When severe, PPE is usually manifested as paresthesias at days 14-21 after the third cycle of chemotherapy, followed by skin eruptions 3-5 days later (22). The syndrome culminates as painful erythema and swelling, predominantly in areas exposed to pressure such as hand palms and feet soles, followed by skin desquamation and re-epithelization. The severity may vary from the development of mild erythema to severe skin damage causing contemporary invalidity of the patient. However, skin affection is temporary with a complete restitution after 2-3 weeks (24). The pathogenesis of PPE remains unknown, but there are several proposed contributing mechanisms. It was proved that anthracyclines and their metabolites are transported to the skin surface by sweat glands (25), these substances then spread laterally and back to the stratum corneum of the epidermis, serving as a reservoir of PLD from which DOX is gradually released (25). A high density of sweat glands and thick stratum corneum are typical of the skin of palms and soles, which may explain the predominant occurrence of PPE in these locations (26).

DOX released from the liposomes is also responsible for the formation of reactive oxygen species (ROS) that induce the production of chemokines and pro-inflammatory cytokines (IL-1 $\beta$, IL-6 a IL-1 $\alpha$ ) in keratinocytes (18). These mediators are responsible for leucocyte chemotaxis and inflammatory reaction enhancement. Moreover, ROS can cause direct damage to collagen fibers. In affected areas, the combination of an inflammatory reaction, keratinocyte's apoptosis and collagen degradation lead to complete skin destruction in the affected areas (27).

Mucositis usually occurs as stomatitis, but rare cases of pharyngo-esophagitis and vulvo-vaginitis were also described (12). The incidence of mucositis is mainly affected by the amount of PLD administered as a single dose, unlike PPE which is associated rather with dose intensity (dose/ time interval between drug administrations) (28). Therefore, dose reduction seems a reasonable approach to reduce the incidence of stomatitis, but has a little impact value in PPE prevention. On the other hand, PPE incidence could be reduced by prolonging the time intervals between individual chemotherapy courses (29). Despite the relatively higher incidence of PPE and mucositis in PLD therapy, the incidence of cardiotoxicity, the major side effect of DOX, is significantly reduced in PLD as well as the incidence of alopecia, myelosupression, nausea, and vomiting (30).

\section{Pharmacokinetics}

The pharmacokinetics of PLD have several properties common in most nanoscale particle based drug delivery systems, which are substantially different from non-liposomal DOX. These properties include a prolonged $\mathrm{T}_{1 / 2}$ of 60-90 hours (PLD doses of 35-70 $\mathrm{mg} / \mathrm{m}^{2}$ (31) as compared to that of DOX with only $20-30 \mathrm{hr} .(32,33,34))$, minimal drug leakage from circulating liposomes, and preferential distribution to the tumor tissue (12). Almost $100 \%$ of the administered dose remains encapsulated in liposomes after intravenous infusion of PLD. These pharmacokinetics are due to the properties of the liposomal carrier, which allows the delivery of the administered drug dose to the target site in encapsulated form. Unlike free DOX, the distribution volume $\left(\mathrm{V}_{\mathrm{d}}\right)$ of PLD is very small and roughly equivalent to the intravascular volume $(35,36)$. The pharmacokinetics of PLD can be described using a one-compartment model with linear pharmacokinetics and proportionally increasing $\mathrm{C}_{\max }$ along with increasing doses $(28,36,37)$. However, according to some authors, the pharmacokinetics of PLD is more accurately modeled as a two-compartment model with an initial $T_{1 / 2}$ of several hours, followed by a slower decrease of PLD concentration with a $T_{1 / 2}$ of 2-3 days, contributing significantly to the AUC $(30,35,38)$. A large diameter of liposomes $(80-90 \mathrm{~nm})$ is responsible for the limited ability of PLD to penetrate through tight endothelial junctions in most capillaries of the human body. Intact endothelium therefore represents a barrier for PLD extravasation into normal tissues and protects them from the toxic effects of DOX (12). As a result of neo-angiogenesis, however, the tumor vasculature is different from that of normal vessels in several aspects. A discontinuous basal membrane, numerous fenestrations and enhanced permeability, enables PLD leakage into the tumor interstitium $(39,40)$. Furthermore, most tumors lack effective lymphatic drainage that could clear PLD from the tumor site resulting in PLD accumulation $(40,41)$. These characteristics are responsible for a phenomenon called enhanced permeation and retention (EPR) effect, which is considered a fundamental principle for the predominant PLD distribution in tumor tissues (also known as passive targeting) $(10,39)$. Maximum PLD concentrations in tumor tissue were detected between 48 and 72 after PLD infusion $(42,43)$. DOX encapsulated in liposomes is gradually released into the tumor tissue resulting in prolonged exposure of cancer cells to the active free drug, which then enters into the cells (30).

RES activity is responsible for the removal of liposomal drug delivery systems from the blood stream, including PLD, by monocytes, macrophages and dendritic cells which are located mainly in the liver, spleen and bone marrow (44). PLD clearance by RES and its factors may also affect the efficacy and toxicity of treatment, otherwise described as pharmacodynamics and toxicodynamics (45). Other factors have been proposed to affect the PLD pharmacokinetics; La-Beck et al. have defined some of them in their retrospective study including 70 patients treated with PLD for solid 
tumors and Kaposi's sarcoma (45). Patients of the male gender, age $<60$ years and higher monocyte count before PLD administration were associated with higher PLD clearance. Pre-cycle monocyte count was also identified as a potential marker of PLD clearance $(45,46)$. A significant decrease in PLD clearance was documented in subsequent chemotherapy cycles (from cycle 1 to cycle 3 ) (45). Further studies will be needed to support these findings, but it is becoming clear that inter-individual variability in the PLD pharmacokinetics represents a serious therapeutic issue as long as it affects both toxicity and treatment outcome $(45,47)$.

\section{Extracorporeal elimination of PLD as a way to reduce toxicity}

Considering the reduced incidence of myelosupression, alopecia, cardiotoxicity, nausea and vomiting, PLD has a more favorable toxicologic profile than conventional forms of DOX (30). However, hand-foot syndrome and mucositis still represent a serious therapeutic issue (21). There are several proposals seeking to reduce these dose and regimen-limiting toxicities, such as the prophylactic use of pyridoxine, avoidance of vasodilatation inducing activities (hot showers, sun exposure), and minimizing skin pressure or friction $(48,49)$.

An innovative and sophisticated approach is based on PLD's unique pharmacokinetics. As mentioned before, the enhanced permeation and retention (EPR) effect is responsible for the predominant and prolonged deposition of PLD in tumor tissues whereas most normal tissues are protected from the toxic effect of PLD thanks to an intact endothelial barrier $(10,39)$. A clinical trial studying the distribution of radiolabeled ( ${ }^{111}$ In-DTPA) pegylated liposomes revealed a high activity in tumor tissue even after 7 days post-administration, whereas $\mathrm{T}_{1 / 2}$ was only of $\sim 76 \mathrm{~h}(50,51)$. The tumor compartment usually represents only a fraction of the whole body compartment, suggesting this is the reason why only a small portion of the total dose (approx. $0.5-3.5 \%$ ) is located within the tumor site despite a relatively selective targeted distribution $(52,51)$. Considering this, along with the fact that PLD concentration in tumor tissue is dependent on actual plasma concentration, it can be presumed that a higher PLD concentration is only needed to build up a relevant diffusion gradient between plasma and tumor compartment (52). The remaining drug pool probably does not have any significant anti-tumor effect after having saturated the tumor tissue, but mainly contributes to adverse effects $(50,52$, 53). It has been shown that the accumulation in the tumor tissue is much faster than that in potential sites of adverse effects (mainly the skin) (54). Charrois et al. studied the pharmacokinetics of PLD in rat models. Tumor tissue $\mathrm{C}_{\max }$ was reached after $24 \mathrm{~h}$, while that in the skin and paws was achieved after $72 \mathrm{~h}(52,54)$. This provides a substantial time gap between the saturation of the tumor site to enable the development of an anti-tumor effect, and critical drug concentration threshold in normal tissues to develop adverse effects (52). The elimination of circulating liposomes within this time interval using a suitable method would very probably prevent PLD accumulation in sensitive tissues and therefore minimize potential adverse effects (52). A greater benefit can be expected in reducing PPE, whereas the ability to reduce hematotoxicity and mucositis will probably be less prominent since these adverse effects are more dependent on $\mathrm{C}_{\max }$ compared with prolonged PLD exposure $(28,52)$.

\section{Extracorporeal apheresis system}

Extracorporeal apheresis is commonly used to eliminate pathogenic substances from the bloodstream. It has been in use for more than three decades to decrease the plasma concentration of LDL (low density lipoproteins) in patients with familiar hypercholesterolemia (55). The fact that LDL and liposomes are similar in several aspects encourages the possibility of eliminating the redundant fraction of circulating liposomes by extracorporeal apheresis systems, of which size exclusion filtration has proved to be the most suitable (56). The usual apheresis technique used in clinical praxis is a double-filtration plasmapheresis (DFPP) (53) since it is able to separate macromolecules from the plasma based on their molecular weight and three-dimensional structure (57). This process is carried out in two main steps: blood cells are separated from plasma in the first step, while in the second step, LDL, liposomes, or other particles with diameter exceeding $30 \mathrm{~nm}$ are separated from plasma (Fig. 3) $(58,62,63)$. The filtration process operates in a continuous fashion and its efficacy depends on the volume of the treated plasma $(53,59)$. The average time for eliminating $60-70 \%$ of circulating LDL is approximately 2-3 hours (53). DFPP is a well tolerated method with minimal, generally mild, side effects (60). Pütz et al. in Freiburg, Germany, were the first to propose the use of extracorporeal apheresis to reduce induced toxicity by nanoscale drug delivery systems (52). Both pre-clinical and clinical trials were performed to prove the efficacy and safety of DFPP in this setting, the results seem promising so far $(53,50)$.

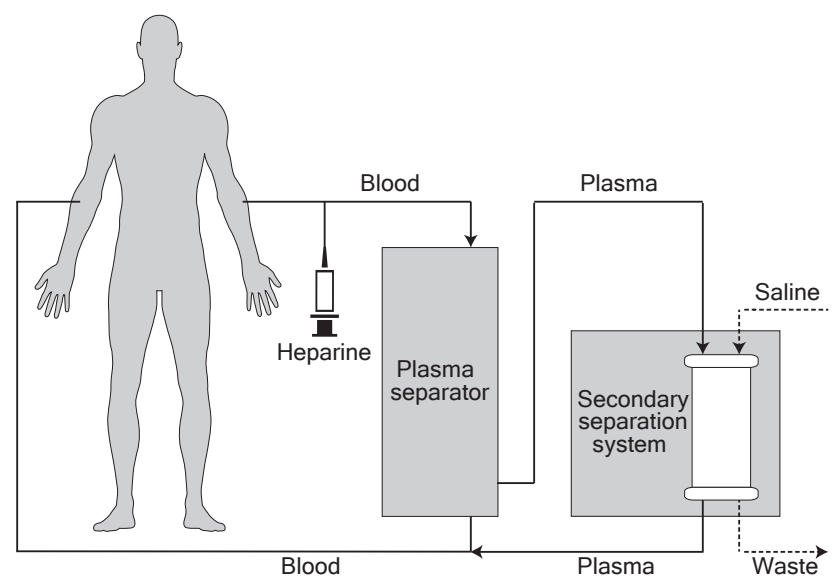

Fig. 3: Schematic diagram of extracorporeal apheresis system. 


\section{First results of PLD extracorporeal elimination to reduce toxicity}

A single clinical trial utilizing extracorporeal elimination of liposomal chemotherapeutics to reduce toxicity has been performed so far, Eckes et al. showed results of the CARL-trial (Controlled Application and Removal of Liposomal chemotherapeutics) in 2011 (50). The study enrolled 15 patients with solid tumors (12 patients with breast cancer treated with PLD/vinorelbine \pm herceptine in the neoadjuvant setting, and 3 patients with recurrent ovarian cancer treated with PLD). The DFPP was performed 42-48 hours after PLD infusion. The safety and efficiency of PLD extracorporeal elimination were addressed as primary endpoints of this study. The DFPP achieved the elimination of approximately $62 \%$ of circulating PLD (corresponding to $\sim 45 \%$ of the initial dose). No relevant DFPP, five grade 2 and one grade 3 treatment related adverse events were reported and only a single case of PPE grade 2 was observed. Reduction of $>30 \%$ in tumor size was achieved in 10 out of 12 breast cancer patients (neoadjuvant) and 1 out of 3 recurrent ovarian patients. It can be presumed that the extracorporeal elimination of PLD using double filtration plasmapheresis is a safe and efficient method to reduce the dose and regimen-limiting toxicity of PLD (50). Our so far unpublished experience with 20 apheresis cycles in metastatic ovarian cancer patients treated with PLD support these findings.

\section{Conclusion}

Neither new chemotherapeutic agents nor targeted therapy (with a few exceptions such as imatinib or trastuzumab), have led to a major improvement in the treatment outcome of solid tumors in past years. The highly selective mechanism of action of targeted therapy seems to be a problem when considering the tumor population's heterogeneity (39). As long as some nanoscale-based drug delivery systems show unique pharmacokinetic features, including selective drug distribution into the tumor tissue regardless of heterogeneity, their use in cancer treatment may help overcome this problem. Nanoscale-based drug delivery systems, including liposomal forms of cytostatic agents, are known to have prolonged $T_{1 / 2}$, and a more convenient distribution pattern and different toxicity profiles (61). Although some adverse effects are less common, others may cause potential dose-limiting toxicity (28). Using an extracorporeal apheresis system could be the solution for reducing these adverse effects without dose reduction or prolonging the time interval between chemotherapy cycles. The indications for currently approved liposomal antineoplastic drugs (e.g. Myocet ${ }^{\circledR}$, Caelyx $^{\circledR}$ ) are broad, and hopefully new nanoscale-based forms of antineoplastic agents will be soon available. Adverse effects similar to those seen in PLD can be expected and extracorporeal apheresis use could be a way to reduce their incidence and severity while achieving the best possible quality of life.

\section{Acknowledgements}

This work was supported by the grant IGA MZ NT 14035-3/2013. Dr. Diaz-Garcia was supported by the Czech Republic State Budget's project no. CZ.1.07/2.3.00/30.0022.

\section{References}

1. Ledermann JA, Raja FA, Fotopoulou C et al. Newly diagnosed and relapsed epithelial ovarian carcinoma: ESMO Clinical Practice Guidelines for diagnosis, treatment and follow-up. Ann Oncol 2013; 24 Suppl 6: vi24-32.

2. Kim A, Ueda Y, Naka T, Enomoto T. Therapeutic strategies in epithelial ovarian cancer. J Exp Clin Cancer Res 2012; 31: 14

3. Fleming GF, Ronnett BM, Seidman J. Epithelial ovarian cancer. In Barakat RR, Markman M, Randall M (eds): Principles and practice of gynecologic oncology, 5th Edition. Philadelphia: Wolters Kluwer Health/Lippincott Williams \& Wilkins 2009; 763-836

4. Scully RE, Sobin LH, Serov SF. Histological typing of ovarian tumours. Berlin, New York: Springer 1999

5. Rubin SC, Randall TC, Armstrong KA et al. Ten-year follow-up of ovarian cancer patients after second-look laparotomy with negative findings. Obstet Gynecol 1999; 93: 21-24

6. Friedlander M, Trimble E, Tinker A et al. Clinical trials in recurrent ovarian cancer Int J Gynecol Cancer 2011; 21: 771-775.

7. National Comprehensive Cancer Network (NCCN): NCCN Clinical Practice Guidelines in Oncology. Ovarian cancer Version 2.2013 (online). Available at: http: //www.ncen.org/professionals/physician_gls/pdf/ovarian.pdf. (3 January 2014, date last accessed)

8. Petruželka L. Pegylovaný lipozomální doxorubicin. Remedia 2008; 18: 407-415.

9. Bozzuto G, Molinari A. Liposomes as nanomedical devices. Int J Nanomedicine. 2015; 10 : 975-99.

10. Maeda H, Wu J, Sawa T et al. Tumor vascular permeability and the EPR effect in macromolecular therapeutics: a review. J Control Release 2000; 65: 271-284.

11. Papahadjopoulos D, Allen TM, Gabizon A et al. Sterically stabilized liposomes: improvements in pharmacokinetics and antitumor therapeutic efficacy. Proc Nat Acad Sci U S A 1991; 88: 11460-11464

12. Soloman R, Gabizon AA. Clinical pharmacology of liposomal anthracyclines: focus on pegylated liposomal Doxorubicin. Clin Lymphoma Myeloma 2008; 8 $21-32$.

13. Caelyx - Product information (online). Date of Most Recent Amendment: 22 October 2013. Available at: http://www.janssen.com.au/files/Products/Caelyx _PI.pdf?cdc150d5ce85dca0aaf4926b5d83144c. (3 May 2014, date last accessed).

14. Green AE, Rose PG. Pegylated liposomal doxorubicin in ovarian cancer. Int J Nanomedicine 2006; 1: 229-239.

15. Minotti G, Menna P, Salvatorelli E et al. Anthracyclines: molecular advances and pharmacologic developments in antitumor activity and cardiotoxicity. Pharmaco Rev 2004; 56: 185-229.

16. Hortobagyi GN. Anthracyclines in the treatment of cancer. An overview. Drugs 1997; 54 Suppl 4: 1-7.

17. Meriweather VD, Bachur NR. Inhibition of DNA and RNA metabolism by daunorubicin and adriamycin in L1210 mouse leukemia, Cancer Res 1972; 32: $1137-42$

18. Sinha BK, Katki AG, Batist G et al. Adriamycin-stimulated hydroxyl radical formation in human breast tumor cells. Biochem Pharmacol 1987; 36: 793-796.

19. Dorr RT. Cytoprotective agents for anthracyclines. Semin Oncol 1996; 23 23-34.

20. Uziely B, Jeffers S, Isacson R et al. Liposomal doxorubicin: antitumor activity and unique toxicities during two complementary phase I studies. J Clin Oncol 1995 13: $1777-1785$.

21. Lotem M, Hubert A, Lyass O et al. Skin toxic effects of polyethylene glycol-coated liposomal doxorubicin. Arch Dermatol 2000; 136: 1475-1480.

22. Gordon AN, Fleagle JT, Guthrie D et al. Recurrent epithelial ovarian carcinoma: a randomized phase III study of pegylated liposomal doxorubicin versus topotecan. J Clin Oncol 2001; 19: 3312-3322.

23. Nagore E, Insa A, Sanmartin O. Antineoplastic therapy-induced palmar planta erythrodysesthesia ('hand-foot') syndrome. Incidence, recognition and management. Am J Clin Dermatol 2000; 1: 225-234.

24. Gabizon AA. Pegylated liposomal doxorubicin: metamorphosis of an old drug into a new form of chemotherapy. Cancer Invest 2001; 19: 424-436.

25. Martschick A, Sehouli J, Patzelt A et al. The pathogenetic mechanism of anthracycline-induced palmar-plantar erythrodysesthesia. Anticancer Res 2009; 29: 2307-2313.

26. Cox GJ, Robertson DB. Toxic erythema of palms and soles associated with highdose mercaptopurine chemotherapy. Arch Dermatol 1986; 122: 1413-1414. 
27. Yokomichi N, Nagasawa T, Coler-Reilly A et al. Pathogenesis of Hand-Foot Syndrome induced by PEG- modified liposomal Doxorubicin. Hum Cell 2013; 26: $8-18$.

28. Lyass O, Uziely B, Ben-Yosef R et al. Correlation of toxicity with pharmacokinetics of pegylated liposomal doxorubicin (Doxil) in metastatic breast carcinoma. Cancer 2000; 89: 1037-1047.

29. Muggia FM, Hainsworth JD, Jeffers S et al. Phase II study of liposomal doxorubicin in refractory ovarian cancer: antitumor activity and toxicity modification by liposomal encapsulation. J Clin Oncol 1997; 15: 987-993.

30. Gabizon A, Shmeeda H, Grenader T. Pharmacological basis of pegylated liposomal doxorubicin: impact on cancer therapy. Eur J Pharm Sci 2012; 45: 388-398.

31. Gabizon A. Applications of liposomal drug delivery systems to cancer therapy. In Mansoor MA (ed) Nanotechnology for Cancer Therapy. New York, USA: CRC Press 2006; 595-611.

32. Greene RF, Collins JM, Jenkins JF et al. Plasma pharmacokinetics of adriamycin and adriamycinol: implications for the design of in vitro experimentsand treatment protocols. Cancer Res. 1983; 43: 3417-21.

33. Rahman A, Carmichael D, Harris M, Roh JK. Comparative pharmacokinetics of free doxorubicin and doxorubicin entrapped in cardiolipin liposomes. Cancer Res. 1986:46: 2295-9.

34. Piscitelli SC1, Rodvold KA, Rushing DA, Tewksbury DA. Pharmacokinetics and pharmacodynamics of doxorubicin in patients with small cell lung cancer. Clin Pharmacol Ther. 1993; 53: 555-61.

35. Gabizon A, Catane R, Uziely B et al. Prolonged circulation time and enhanced accumulation in malignant exudates of doxorubicin encapsulated in polyethylene-glycol coated liposomes. Cancer Res 1994; 54: 987-992.

36. Fujisaka Y, Horiike A, Shimizu T et al. Phase 1 clinical study of pegylated liposomal doxorubicin (JNS002) in Japanese patients with solid tumors. Jpn J Clin Oncol 2006; 36: 768-774.

37. Amantea M, Newman MS, Sullivan TM et al. Relationship of dose intensity to the induction of palmar-plantar erythrodysesthia by pegylated liposomal doxorubicin in dogs. Hum Exp Toxicol 1999; 18: 17-26.

38. Amantea MA, Forrest A, Northfelt DW, Mamelok R. Population pharmacokinetics and pharmacodynamics of pegylated-liposomal doxorubicin in patients with AIDS-related Kaposi's sarcoma. Clin Pharmacol Ther 1997; 61: 301-311.

39. Fang J, Nakamura H, Maeda H. The EPR effect: Unique features of tumor blood vessels for drug delivery, factors involved, and limitations and augmentation of the effect. Adv Drug Deliv Rev 2011; 63: 136-151.

40. Matsumura Y, Maeda H. A new concept for macromolecular therapeutics in cancer chemotherapy: mechanism of tumoritropic accumulation of proteins and the antitumor agent smancs. Cancer Res 1986; 46: 6387-6392.

41. Maeda H. SMANCS and polymer-conjugated macromolecular drugs: advantages in cancer chemotherapy. Adv Drug Deliv Rev 2001; 46: 169-185.

42. Vaage J, Barbera-Guillem E, Abra R et al. Tissue distribution and therapeutic effect of intravenous free or encapsulated liposomal doxorubicin on human prostate carcinoma xenografts. Cancer 1994; 73: 1478-1484.

43. Gabizon A, Goren D, Horowitz AT et al. Long-circulating liposomes for drug delivery in cancer therapy: a review of biodistribution studies in tumor-bearing animals. Adv Drug Deliv Rev 1997; 24: 337-344.

44. Van Rooijen N. The Liposome-Mediated "Macrophage Suicide" Technique: A Tool to Study and Manipulate Macrophage Activities. In Gregoriadis G (ed) Liposome Technology, 3rd Edition. New York, USA: Informa Healthcare 2006; 303-315.
45. La-Beck NM, Zamboni BA, Gabizon A et al. Factors affecting the pharmacokinetics of pegylated liposomal doxorubicin in patients. Cancer Chemother Pharmacol 2012; 69: 43-50.

46. Gabizon A, Isacson R, Rosengarten O et al. An open-label study to evaluate dose and cycle dependence of the pharmacokinetics of pegylated liposomal doxorubicin. Cancer Chemother Pharmacol 2008; 61: 695-702.

47. Ko EM, Lippmann Q, Caron WP et al. Clinical risk factors of PEGylated liposomal doxorubicin induced palmar plantar erythrodysesthesia in recurrent ovarian cancer patients. Gynecol Oncol 2013; 131: 683-688.

48. Edwards SJ. Prevention and treatment of adverse effects related to chemotherapy for recurrent ovarian cancer. Semin Oncol Nurs 2003; 19: 19-39.

49. Vail DM, Chun R, Thamm DH et al. Efficacy of pyridoxine to ameliorate the cutaneous toxicity associated with doxorubicin containing pegylated (Stealth) liposomes: a randomized, double-blind clinical trial using a canine model. Clin Cancer Res 1998; 4: 1567-1571.

50. Eckes J, Schmah O, Siebers JW et al. Kinetic targeting of pegylated liposomal doxorubicin: a new approach to reduce toxicity during chemotherapy (CARL-trial). BMC Cancer 2011; 11: 337

51. Harrington KJ, Mohammadtaghi S, Uster PS et al. Effective targeting of solid tumors in patients with locally advanced cancers by radiolabeled pegylated liposomes. Clin Cancer Res 2001; 7: 243-254.

52. Putz G, Schmah O, Eckes J et al. Controlled application and scheduled removal of nanoparticle based chemotherapeutics (CARL) will reduce dose limiting adverse events in anticancer chemotherapy. Med Hypotheses 2009; 72: 393-397.

53. Putz G, Schmah O, Eckes J et al. Controlled application and removal of liposomal therapeutics: effective elimination of pegylated liposomal doxorubicin by double-filtration plasmapheresis in vitro. J Clin Apher 2010; 25: 54-62.

54. Charrois GJ, Allen TM. Multiple injections of pegylated liposomal Doxorubicin: pharmacokinetics and therapeutic activity. J Pharmacol Exp Ther 2003; 306: 1058-1067.

55. Borberg H. 26 years of LDL-apheresis: a review of experience. Transfus Apher Sci 2009; 41: 49-59.

56. Putz G, Eckes J, Schmah O et al. Elimination of liposomes by different separation principles used in low-density lipoprotein apheresis. Ther Apher Dial 2008; 12 . $2-12$.

57. Klingel R, Fassbender T, Fassbender C, Gohlen B. From membrane differential filtration to lipidfiltration: technological progress in low-density lipoprotein apheresis. Ther Apher Dial 2003; 7: 350-358.

58. Takada Y, Ito T, Ueda Y et al. Effects of double-filtration plasmapheresis combined with interferon plus ribavirin for recurrent hepatitis $\mathrm{C}$ after living donor liver transplantation. Liver Transpl 2008; 14: 1044-1047.

59. Bosch T. Therapeutic Apheresis-State of the Art in the Year 2005. Ther Apher 2005; 9: 459-468.

60. Malchesky PS, Koo AP, Roberson GA et al. Apheresis technologies and clinical applications: the 2002 international apheresis registry. Ther Apher Dial 2004; 8 : 124-143.

61. Allen TM, Cullis PR. Drug delivery systems: entering the mainstream. Science 2004; 303: 1818-1822.

62. Blaha M, Rencova E, Langrova $\mathrm{H}$ et al. Rheohaemapheresis in the treatment of nonvascular age-related macular degeneration. Atheroscler Suppl. 2013; 14: 179-84.

63. Blaha M, Rencova E, Blaha V et al. The importance of rheological parameters in the therapy of microcirculatory disorders. Clin Hemorheol Microcirc. 2009; 42: $37-46$.

\section{Corresponding author:}

Ondřej Kubeček, Department of Oncology and Radiotherapy, Charles University in Prague, Faculty of Medicine and University Hospital in Hradec Králové, Sokolská 581, 50005 Hradec Králové, Czech Republic; e-mail: okubec@gmail.com 